\title{
La herencia en las empresas familiares de la región pampeana argentina durante el actual período de auge económico de la actividad agrícola
}

\author{
Melina Neiman \\ Centro de Estudios e Investigaciones Laborales (CEIL) / CONICET \\ mneiman@ceil-conicet.gob.ar
}

Recepción: 30-11-2015

Aceptación: 10-09-2016

\section{Resumen}

El artículo se propone mostrar la evolución reciente de los modelos de sucesión hereditaria de las unidades agropecuarias en un área llamada «zona núcleo» de la región pampeana argentina, que durante la década pasada y buena parte de la actual, atraviesa por un período de auge económico impulsado por el aumento en el precio de los productos básicos en el mundo, principalmente la soja.

Se analizan los procesos hereditarios en la agricultura familiar mostrando los arreglos que se establecen entre generaciones y géneros para la gestión de la empresa, previo al reparto definitivo de la propiedad entre los herederos.

El abordaje metodológico es principalmente cualitativo, basado en entrevistas en profundidad a productores familiares, hijos, hijas y esposas de productores del partido de Junín, en la provincia de Buenos Aires. Se recurre, además, a fuentes secundarias para considerar los factores relacionados con la expansión productiva de la agricultura y con el precio de la tierra en el área de estudio, así como con información provista por profesionales vinculados con el tema (abogados, escribanos y contadores).

Palabras clave: herencia; familias; agricultura; sojización; región pampeana argentina

\section{Abstract. Inheritance Among Family Farms of Argentina's Pampas During the Current Economic Boom of Agriculture}

The article aims to show the current hereditary succession models among family farms in the so-called "core area" of Argentina's Pampas. The region has experienced a period of economic boom over the past decade and part of the current one driven by worldwide increases in commodity prices, mainly soybean. Inheritance processes are analyzed to determine the arrangements between generations and genders concerning farm management prior to the final distribution of property among heirs. The methodology is mainly based on in-depth interviews with family farmers, and their sons, daughters and wives at 
Junín in the province of Buenos Aires. Secondary sources of information are also used in order to examine factors related to the expansion of agriculture production and land price increases in the study area, as well as data provided by professionals such as lawyers, notaries and accountants.

Keywords: inheritance; families; agriculture; soybean; Argentina’s Pampas

\section{Sumario}

1. Introducción 5. Los arreglos intrafamiliares en los procesos sucesorios

2. Modelos de sucesión, procesos hereditarios y arreglos familiares

6. Conclusiones

3. Metodología

Referencias bibliográficas

4. El precio de la tierra y la renta agraria en la región pampeana

\section{Introducción}

La herencia ha sido considerada tradicionalmente como una institución que se vale de estrategias de distinto tipo para preservar la familia, el patrimonio y la propiedad común. Adquiere una significación particular en los casos en que los miembros tienen una alta dependencia de los ingresos provenientes de la empresa parental, como cuando el propósito es conservar la fortuna familiar que la generación anterior ha logrado acumular.

Los temas relacionados con la herencia de la propiedad y el patrimonio durante la transición generacional han sido motivos de debate en las ciencias sociales en general y en la sociología rural en particular. En este caso, tanto para la agricultura de los países avanzados como para la de las regiones en desarrollo.

La perspectiva de análisis centrada en la relación que se establece entre el desenvolvimiento de "la familia» como institución y el desarrollo del capitalismo tuvo su apogeo desde fines del siglo XIX, cuando se sostenía que «la familia era el corazón de la empresa capitalista» (Colli y Rose, 1995). Las empresas familiares eran las unidades de producción y acumulación, y tenían el «orgullo de que el dueño de la empresa era conocido por el apellido de la familia». La sucesión era guiada por la continuidad parental, con lo que se creaban «dinastías a través de las generaciones», por ello se conceptualizaba esta etapa en términos de "capitalismo familiar» (Colli et al., 2003; Colli y Rose, 1995).

En la primera posguerra, el clásico libro de Berle y Means (1932) afirma la «irrelevancia de la familia» en la organización de las clases capitalistas. Argumentan que se separa la propiedad de la gran empresa de su control porque la persona corporativa, que era formalmente dueña de una entidad corporativa, se convierte en un accionista más y se contratan gerentes que toman el control de las actividades de las compañías. De esta forma, la familia se vuelve un anacronismo. 
En los años ochenta, esta tesis gerencial es fuertemente criticada. Así, distintos autores neomarxistas (Zeitlin, 1989; Dunn, 1980) muestran la persistente influencia familiar en los niveles más altos de los negocios y de la sociedad, donde los grupos familiares y las empresas son nodos de "formas de organización en red» y las "esferas familiares de influencia» operan en y entre las empresas (Gilding, 2005).

Este artículo se propone comprender el modelo de sucesión resultante de la combinación de los diferentes arreglos familiares que se producen a lo largo del proceso hereditario de las unidades agropecuarias de la llamada "zona núcleo» ${ }^{1}$ de la región pampeana, en el actual período de auge económico. Se trata de pequeñas y medianas empresas gestionadas por un productor que, a su vez, está condicionado por los vínculos de sangre, tanto en el momento de tomar decisiones como de distribuir ganancias u organizar el traspaso generacional de la gestión de la explotación y de la propiedad de la tierra.

En la región pampeana argentina, los procesos hereditarios experimentaron importantes transformaciones en un período de crecimiento económico impulsado por el aumento en el precio de los productos básicos. Las pequeñas y medianas empresas a las que se refiere este artículo atraviesan problemáticas similares en cuanto a la preocupación por la preservación del control de la gestión y la continuidad familiar de las unidades. Esto se pone de manifiesto en los modelos de herencia preponderantes y en las tensiones que aparecen en relación con los procesos de individuación de los miembros de las familias, lo cual da lugar a diferentes conflictos y arreglos intrafamiliares a lo largo del proceso sucesorio ${ }^{2}$.

A su vez, estas empresas familiares se vieron transformadas por un contexto particular marcado, por un lado, por el avance de un patrón productivo basado en la intensificación de la producción, lo que provocó un extraordinario aumento de la renta y de la tierra en propiedad y, por otro lado, por los cambios sociales y demográficos presentes en las dinámicas familiares en lo que se refiere centralmente al vínculo entre géneros y generaciones en el interior de las familias.

En este artículo, primero, se recuperan los antecedentes conceptuales acerca de los diferentes modelos de herencia, los procesos de sucesión y la multiplicidad de arreglos existentes para que se produzca la transición generacional; posteriormente, se expone un apartado metodológico en donde se detalla la forma en que se llevó a cabo el proceso de investigación; luego, se presentan las principales transformaciones propias del proceso de agriculturización en curso, que atravesó la región y que llevaron a que se incrementaran los precios de la tierra. Finalmente, se analizan específicamente los procesos hereditarios

1. Se denomina «zona núcleo» de la Región Pampeana a los partidos que, por las características de sus suelos y climas, presentan las mejores condiciones para el desarrollo de cultivos como los cereales y las oleaginosas.

2. Los temas relacionados con el conflicto en torno a la sucesión intergeneracional ya se encuentran presentes en artículos de la Harvard Business Review de 1960 y 1970 (Donnelley, 1964; Levinston, 1971). 
poniendo especial atención en comprender los arreglos que se establecen para la gestión de la empresa familiar previos al reparto definitivo de la propiedad entre los herederos.

Entre los principales hallazgos de este artículo, se muestra, por un lado, que los procesos hereditarios comienzan mucho antes del retiro del productor agropecuario de la unidad productiva y del relevo generacional definitivo. En este período, se ponen en juego distintos tipos de arreglos intrafamiliares para compartir la gestión de la producción y repartir las ganancias de la empresa agraria. Por otro lado, se destaca la importancia de los altos niveles de la renta de la tierra y de la rentabilidad de la producción para considerar a todos los herederos en la estrategia sucesoria, incluyendo a las hijas mujeres y a aquellos hijos que se retiraron de la actividad para seguir su propia trayectoria profesional, pero que pueden, en un futuro, regresar a la empresa atraídos por los altos ingresos. Se conforma así un modelo de sucesión a partir de la combinación de arreglos económicos y no económicos que se corresponden con criterios afectivos y parentales que apunta a mantener a la empresa familiar en funcionamiento, pero también para responder a los proyectos de vida de los miembros del hogar.

\section{Modelos de sucesión, procesos hereditarios y arreglos familiares}

Los procesos de sucesión han sido estudiados durante las últimas décadas desde diferentes perspectivas que se preocuparon por analizar dinámicas presentes en modelos distintos de hogares. Se pueden distinguir, analíticamente, investigaciones que buscan definir los modelos de sucesión como perspectiva que señala, de manera holística, las «reglas» que se instrumentan en el relevo generacional; otras apuntan a describir y a analizar cómo se atraviesan las distintas etapas de los procesos hereditarios, y también están aquellas que ponen énfasis en entender la multiplicidad de arreglos familiares que se establecen para resolver los conflictos y cumplir con la transición generacional.

Cuando se construyen modelos de sucesión, se busca mostrar las reglas informales que siguen las familias para establecer el reparto del patrimonio entre los hijos y las hijas en el momento del relevo generacional. Las familias tienen reglas para elegir al sucesor (Rogers y Salomon, 1983; Keating y Munro, 1989), incluso aunque esas reglas no sean explícitas. Estas incluyen decisiones vinculadas con el número de sucesores, su género, el orden de nacimiento, su dedicación y sus habilidades personales para la gestión de la empresa y para el trabajo agropecuario.

Keating y Little (1997) muestran que el género es el criterio más importante para definir quién será el sucesor y que, si bien la regla de que «las mujeres no pueden ser sucesoras» fue rechazada en el discurso por los distintos productores considerados en su estudio, esta sigue siendo preponderante. Las mujeres generalmente no son percibidas como sucesoras elegibles, y más en los casos en que hay hijas mujeres e hijos varones (Bennet, 1982; Keating y Little, 1997). Sin embargo, Gasson et al. (1988) sugieren que existen actitudes 
tendientes a la equidad de género que hacen que las mujeres se vuelvan elegibles como sucesoras. Gilding (2005) también muestra que, de las doscientas familias más ricas de Australia, «el 93\% de los respondientes sostienen el principio de reparto igualitario de la herencia entre sus hijos, incluyendo las hijas» (Gilding, 2005: 39).

Los modelos de herencia también hacen referencia a cómo se planifica esta etapa en el interior del hogar. Anderson y Rosenblatt (1985) encuentran que muchas familias agrícolas en Estados Unidos no se sienten obligadas a explicitar su estrategia hereditaria o a hablar con sus hijos de sus opciones de carrera o de sus propios planes de retiro. Sin embargo, las decisiones vinculadas con la sucesión tienen importantes ramificaciones para el futuro de la empresa agropecuaria, y estas decisiones se vuelven complejas en los períodos de suba del precio de la tierra, de volatilidad de los mercados y de futuro incierto de la agricultura. Distintos estudios indican que, cuando hay una comunicación más abierta sobre la distribución futura de la herencia, la intensidad del desacuerdo sobre los temas es menor (Danes et al., 2000). Asimismo, cuando no se tiene la oportunidad de discutir ni de tomar decisiones sobre los temas de la empresa familiar, el conflicto tiende a intensificarse (Stewart y Danes, 2001; Danes y Lee, 2004).

Para el caso específico de la región pampeana argentina, Muzlera (2009) sostiene que la modalidad de herencia posee características propias basadas en la idea de que la herencia es "cosa de hombres», tanto en el plano material (tierras, inmuebles, etc.) como en el simbólico (identidad chacarera).

La herencia funciona así no sólo como mecanismo de reproducción social, sino también como núcleo de prácticas de dominación masculina: la herencia paterna es la más importante y si hubiese que optar entre vender un campo u otro, el originado en la herencia paterna es el que se busca preservar con más ahínco. La tierra es asociada al apellido y éste es trasmitido patrilinealmente. (Muzlera, 2009: 73)

De este modo, argumenta que la herencia es un modo de vida que se busca preservar, y el hecho de que este vínculo patrilineal se conserve asegura la sobrevivencia de la figura del agricultor familiar.

Los estudios que enfocan cómo se produce la transición de este proceso hereditario muestran que la sucesión está vinculada con el ciclo vital de las familias, durante el cual la unidad económica también atraviesa una serie de etapas. Los mencionados Anderson y Rosenblatt (1985) afirman que el proceso de sucesión comienza cuando el último hijo está en preescolar o en los primeros grados y termina recién cuando el sucesor se hace cargo del control de la gestión de la unidad y la generación anterior se retira. En este mismo sentido, Hutson (1987) explica que el proceso sucesorio puede ser dividido en cuatro fases:

- El escalón inicial, cuando los hijos asisten a la escuela como actividad principal y comienzan a trabajar bajo la supervisión del padre. 
— La segunda fase, cuando padres e hijos deciden expandir e intensificar la producción.

- La tercera fase ocurre cuando se lleva a cabo la implementación de nuevos esquemas o métodos en los cuales son delegadas a los hijos las responsabilidades del negocio familiar.

- Finalmente, la cuarta fase corresponde al momento en que el padre se retira y la gestión de la explotación es completamente transferida a la generación siguiente.

Con respecto a dichos sistemas de reparto de la herencia que se definen en este proceso, Wolf (1978) señaló en los años sesenta que la ideología de la sucesión está fundamentada en ciertas normas que regulan la manera en que se transfiere el control de la tierra. Menciona que existen dos sistemas hereditarios: el de la herencia no divisible, basado en la existencia de un único heredero designado por el jefe de la unidad familiar, y el de la herencia divisible, en la que hay más de un sucesor y la tierra se divide entre las partes.

Burton y Walford (2005) han analizado que existen tres opciones principales para el desarrollo del proceso sucesorio. En la primera, la división de la tierra incluye a hermanos que ya no están en el campo, lo cual no significa necesariamente que se subdivida la unidad original. Los arreglos de renta son relativamente comunes y, cuando la tierra es vendida por el sucesor «no rural», puede que sea comprada directamente por el agricultor original. Se trata, esencialmente, de una simple transferencia de capital de la empresa agropecuaria a uno de los hermanos. Asimismo, cuando el sucesor no tiene el capital necesario para realizar esta operación y pagarle a su hermano, la fracción de tierra respectiva puede ser vendida. En el segundo caso, el predio puede ser dividido «temporalmente», con la intención de realizar una reintegración posterior. La tercera opción es que las «nuevas unidades» se manejen de forma individual por parte de los hermanos, ya sea por efecto de un conflicto intrafamiliar o por una división de mutuo acuerdo. A estos sistemas más tradicionales de la herencia, se suman las estrategias tendientes a priorizar la «movilidad de los descendientes» mediante el acceso a la educación frente a las estrategias que garantizan la reproducción del patrimonio familiar (González y Gómez Benito, 1997).

Para analizar las dinámicas propias que presenta la forma en que se produce el relevo sucesorio en la región pampeana, Bardomás (2000), en su trabajo sobre la agricultura familiar de Pigüé, en la provincia de Buenos Aires, sostiene que

[...] la transferencia de tierras se arregla entre familiares que se retiran de la actividad y sus sucesores que ingresan a ella sin que medie la participación del mercado. Así, en cierta manera, se conforma un mercado diferente, basado en la familia, con particularidades y con leyes distintas a las de la oferta y la demanda. (Bardomás, 2000: 11)

Asimismo, argumenta que los cambios que pueden producirse en torno a las estrategias sucesorias se encuentran relacionados con características de 
las estructuras familiares y con decisiones tomadas por los propios productores, en las cuales los determinantes económicos representan solo uno de los aspectos.

Si bien el «buen proceso» del relevo generacional se presenta como un «deseo natural» de las familias (Gilding, 2005), no siempre se logra exitosamente. En principio, las etapas del ciclo vital parental (Chayanov, 1975; Fortes, 1958; Robichaux, 2007) condicionan las fases del proceso sucesorio de la empresa familiar capitalista; además, la definición misma de la empresa familiar cambia, tanto al atravesar el ciclo vital de los hogares como por el devenir de los contextos político-económicos donde se desenvuelven.

Los conflictos y los posteriores arreglos familiares para ordenar la sucesión de una empresa familiar aparecen porque involucran relaciones interpersonales sensibles. Esto incluye disputas de poder entre padres e hijos o hijas, así como posibles rivalidades entre hermanos y/o hermanas que ocurren ante el desafío de manejar el cambio. Los conflictos pueden estar relacionados con una noción de justicia, ya que se refieren a problemas de compensación e igualdad de trato vinculados con la distribución de los recursos (McClendon y Kadis, 1991). En una empresa agrícola, las actividades deben ser coordinadas y reguladas para asegurar la estabilidad de la unidad económica, específicamente quién hace qué, cómo son distribuidos los recursos, quién toma las decisiones y sobre qué temas decide (McCullom, 1988; Rettig et al., 1999), y estas decisiones generan tensiones en momentos de cambio como lo es la sucesión generacional. Este tránsito implica cambios junto con la necesidad de que sean lo menos traumáticos posible para que el negocio familiar continúe siendo viable, aunque las tensiones y los conflictos sean una parte natural de este proceso (Danes y Amarapurkar, 2001). Incluso tolerar ciertos niveles de conflicto puede estimular el cambio que se necesita dar, aunque el conflicto también puede volverse destructivo en cierto punto (Danes y Olson, 2003). Abramovay (2001), para el caso de la agricultura familiar en Santa Catarina (Brasil), explica que el proceso sucesorio puede conducir a conflictos que van desde la forma de remuneración de los hermanos no contemplados en la herencia paterna, hasta cuestiones de género. Además, esta etapa se encuentra signada por las obligaciones familiares con respecto al sostenimiento económico de las personas mayores, particularmente importante en los países que no han desarrollado un estado de bienestar fuerte (Cooney y Dykstra, 2011; Keating et al., 2014).

De esta manera, se produce una demora en la definición de los arreglos familiares, que involucran tanto al heredero y a la continuidad de la unidad de producción paterna como al destino de los demás hermanos no sucesores. En esta etapa, se pone en discusión la necesidad de definir el valor de la propiedad y las formas de recompensa a los hijos no contemplados en la herencia. Sin embargo, sostienen Nosé et al. (2015) que las familias que hoy tienen un razonable nivel de diálogo sobre el destino de los hijos y sobre la organización de la propiedad, difícilmente lleguen a abordar los temas de naturaleza sucesoria de una manera conflictiva. Por otro lado, Barthez (1990) y Baptista (1995) coinciden en afirmar que el desenlace de trayecto- 
rias individuales por parte de los diferentes miembros contribuye a la posible ruptura de la unidad entre familia y explotación y, de este modo, incorpora otro foco de conflicto.

$\mathrm{Al}$ analizar dinámicas propias de la región pampeana, Craviotti (2001) retoma trabajos de Archetti y Stölen (1975) y Jervell (1999) para mostrar que, en las explotaciones familiares, tanto la toma de decisiones como el manejo de conflictos internos presentan rasgos propios, por ser empresas y grupos domésticos a la vez, y que la designación del sucesor es un conflicto estructural básico. Vinculado a ello, están las estrategias hereditarias que, en distintos análisis, se revelan como tendientes a preservar la explotación desde el punto de vista productivo (Archetti y Stölen, 1975; Jervell, 1999), lo que muestra que existe una asociación entre herencia y aportes efectuados por los hijos en materia de dedicación a la explotación. Asimismo, si el traspaso se efectúa en vida de los padres, esto supone ciertas obligaciones en términos de responder por su manutención (Craviotti, 2001).

Gras (2010) destaca que la herencia no contiene, como sí era antes, la transmisión conjunta de la propiedad de la tierra y del oficio a las nuevas generaciones. Si bien el mecanismo hereditario no estuvo nunca exento de tensiones y contradicciones, su doble dimensión — la transmisión de la tierra y de un modo de gestionarla, a partir de la dedicación del heredero a un oficio específico- intervenía fuertemente en la reproducción intergeneracional de la agricultura familiar. Sin embargo, en el actual escenario, la herencia de la tierra se ve separada de la herencia de la profesión (Gras, 2010).

En resumen, las investigaciones que apuntaron a la producción de modelos de sucesión señalan cuáles son las variables consideradas para establecer un "criterio hereditario» y analizan el grado de conocimiento del mismo. Entre las principales variables, se reconoce el género, la dedicación a la actividad y el orden de nacimiento, entre otras. Asimismo, se explica que existe una asociación directa entre la visibilidad de las mismas y la posibilidad de disminuir los niveles de conflicto intrafamiliar.

Otra perspectiva para analizar la herencia se enfoca en mostrar la transición de este proceso a través del estudio de las trayectorias parentales. Desde este punto de vista, las unidades productivas atraviesan una serie de etapas que están condicionadas por el ciclo vital de las familias y por la necesidad de garantizar la reproducción del patrimonio familiar. Estos procesos se desenvuelven en determinados contextos político-económicos que también inciden en la posibilidad de tener éxito en el relevo generacional y en el mantenimiento de la unidad.

El análisis en torno a los arreglos familiares busca entender cómo se actúa para la resolución de los conflictos producto de la distribución de la herencia. Estos arreglos responden a demandas económicas, pero también afectivas, por tratarse de empresas cuyos miembros, además, tienen relaciones parentales. Entre los arreglos existentes, se cuenta con diferentes modos de reparto del patrimonio y de las ganancias, pero también suponen obligaciones para los herederos. 


\section{Metodología}

El trabajo de campo se llevó a cabo en el partido de Junín, que se ubica en el noroeste de la provincia de Buenos Aires y presenta unas características clave en cuanto a la actividad cerealera y oleaginosa practicada por unidades familiares y empresariales. En efecto, el desarrollo agrícola en el partido se ve favorecido por las condiciones naturales del suelo y del clima, características que lo convierten en una de las zonas más productivas del país, dominado en los últimos años por el llamado "proceso de sojización»"

El abordaje metodológico es principalmente cualitativo y se basa en la realización y el análisis de entrevistas en profundidad. Se llevaron a cabo treinta entrevistas en profundidad a titulares de explotaciones familiares, hijos, hijas y esposas de productores del partido de Junín. Se recurre, además, a fuentes secundarias para explicar los factores relacionados con la expansión productiva de la agricultura y con el precio de la tierra. Todo ello se complementa con cuatro entrevistas a informantes clave, como profesionales vinculados a estos temas (escribano, operador inmobiliario, asesor agropecuario, contador).

El criterio para seleccionar a las personas entrevistadas es conceptualmente intencionado. Se busca examinar patrones de similitudes y diferencias en un número moderado de casos y se intenta captar y comprender su diversidad. Este enfoque, que apunta a comparar los casos entre sí, es especialmente adecuado para explicar la diversidad, interpretar la relevancia cultural e histórica de un hecho y hacer progresar la teoría (Ragin, 2007).

La realización de entrevistas a diferentes miembros de los hogares permitió, a través de la comparación de experiencias, responder las preguntas de la investigación en que se fundamenta este artículo. El procedimiento llevado a cabo para la selección de entrevistados se basó en la consideración de un concepto amplio de empresa agrícola familiar, buscando que primaran explicaciones que dieran cuenta de la heterogeneidad de este universo antes de que delimitaran analíticamente esta categoría. La técnica de selección utilizada fue la denominada «bola de nieve», que permitió establecer el contacto con distinto tipo de personas entrevistadas según la etapa de investigación.

El trabajo de campo estuvo organizado en cuatro visitas en las que se realizaron las entrevistas en profundidad que permitieron avanzar de manera sistemática en la recolección de información y la producción del conocimiento. Las primeras etapas se remontan al año 2012. En mayo de ese año, se entrevistaron a cuatro productores agropecuarios y a cuatro esposas de productores, y en agosto de ese mismo año, se pudieron llevar a cabo otras ocho entrevistas a productores agropecuarios y seis entrevistas a hijos varones de productores. Para abril de 2013, se decidió incorporar testimonios de mujeres, por lo que se entrevistó a ocho hijas de productores agropecuarios. Finalmente, en junio

3. En el período 2013-2014, el cultivo de la soja ocupa aproximadamente 157.000 hectáreas (MINAGRI, 2016), mientras que, en 2002, la superficie implantada alcanzaba las 66.892 hectáreas y, en 1988, solo 30.008 hectáreas (INDEC, 1988 y 2002). 
de 2014, se realizó la última etapa de trabajo de campo, pero esta vez con profesionales que desarrollaban distintas actividades relacionadas con la temática de este artículo: se entrevistaron a dos operadores inmobiliarios, un escribano y un contador que trabajaba como asesor agropecuario.

A partir de la información producida por las entrevistas en profundidad, se comenzaron a buscar los ejes centrales que atravesaban a las mismas. Mediante la codificación de las entrevistas, se explicaron procesos y se construyeron conceptos capaces de explicar los arreglos familiares que se originan como parte constitutiva del proceso sucesorio. El análisis categorial realizado permite delimitar dimensiones y variables con el objetivo de organizar la información sin perder la heterogeneidad de respuestas ni de perspectivas. En esta investigación, los principales ejes de análisis estuvieron vinculados con las formas de organización del trabajo y la producción, el lugar ocupado por el trabajo familiar, el papel del trabajo no familiar, los ciclos parentales, los arreglos entre padres e hijos (para la toma de decisiones y la distribución de ganancias), las trayectorias individuales de los miembros de los hogares y las relaciones de género.

\section{El precio de la tierra y la renta agraria en la región pampeana}

Los procesos hereditarios en estudio se encuentran situados en contextos de cambios económicos y sociales que afectaron al precio de la tierra, al valor de los arrendamientos y a la rentabilidad de la producción.

La renta de la tierra hace referencia a la forma de valorización de la misma ${ }^{4}$, donde esta aparece con su precio, que luego impacta sobre el cálculo de los precios de las mercancías agrícolas. Estos precios, a su vez, deben mantener una correspondencia con la productividad social del sector, por lo que el valor excedente producido no puede ser menor que la suma de las ganancias, de la renta y del interés apropiados (Valle Baeza, 1991).

Los arreglos sucesorios de los pequeños y medianos productores se encuentran condicionados por un contexto general de incremento de la demanda por tierras y de altos precios de los productos básicos, pero también por transformaciones familiares en lo referido, por ejemplo, a la individuación de las trayectorias profesionales de sus miembros.

En este sentido, se busca comprender cómo este marco de relaciones impacta sobre la forma en que se establecen los vínculos parentales en relación con

4. En la discusión acerca de la naturaleza de la tierra como factor de producción, Marx (1976) y Kautsky (1978) coinciden en que la tierra es un recurso fijo en cuanto a su cantidad y que representa una forma relativamente inamovible del capital. La tierra no puede ser socialmente creada ni multiplicada, ni tampoco puede ser transportada a locaciones más adecuadas para incrementar la producción y las ganancias (Mann, 1990). En palabras de Marx (1976), la tierra es un medio de producción no reproducible, lo que presupone un monopolio «de ciertas personas que les da derecho a disponer sobre determinadas porciones del planeta como esferas privativas de su voluntad propia, con exclusión de todo lo demás. Partiendo de eso, se trata de explotar el valor económico, es decir, de valorizar este monopolio a base de la producción capitalista» (Marx, 1976: 574). 
el proceso hereditario. Para ello, se presentan algunas tendencias comunes que dan cuenta del contexto general de aumento de precios de la producción agrícola y de la renta de la tierra para la región pampeana, lo cual genera mayores ingresos que después son apropiados de distintas formas (arrendamiento, producción, retenciones) por parte de los diversos actores sociales, principalmente, los propietarios de la tierra y los productores.

Este enfoque permitirá observar posteriormente cómo se expresan estas disputas por la apropiación de los ingresos — ganancia, renta y salario— hacia el interior de las familias y de las unidades productivas en un momento crítico como lo es la transmisión de la propiedad y de la gestión de la explotación.

El precio de las mercancías producidas en la región estudiada ha experimentado un fuerte incremento en el mercado mundial, empujado por la incorporación de grandes contingentes de población —en tanto consumidores - al mercado alimentario. Por ejemplo:

[...] en los 14 años comprendidos entre 1994 y 2007, China pasó de comprar en los mercados internacionales 52 mil toneladas de porotos de soja a casi 31 millones de toneladas, con lo que incrementó la cifra inicial 597 veces. Argentina acompañó esta expansión y, para 2007, cubrió aproximadamente el 30\% de las importaciones chinas totales de este producto, por detrás de Brasil y Estados Unidos. (Keller, 2008: 1)

También se han señalado algunos movimientos financieros especulativos que pudieron haber actuado en la misma dirección.

En el caso particular de la producción de soja, se puede observar que su precio en dólares se ha multiplicado por cinco durante el período 2003-2013. Asimismo, se encuentra la misma tendencia hacia el alza de precios en el caso de otros cultivos como el maíz, el arroz y el trigo (MINAGRI, 2016).

La renta de la tierra como parte constitutiva del precio de la mercancía agrícola también se ha incrementado de forma paralela al aumento del precio de los productos. En el caso de Junín — una zona agrícola maicera-, hace unos diez años, el precio de la tierra era de 2.500 dólares la hectárea y actualmente es de 15.000 dólares (Márgenes Agropecuarios). Un operador inmobiliario de la zona confirma que «el valor promedio del precio de la tierra en la región aumentó alrededor del 400 por ciento en los últimos diez años y se ubica en unos 15.000 dólares por hectárea, aunque en algunos distritos trepa hasta los 20.000 dólares» (La Democracia, 2011).

De cualquier manera, no necesariamente se concretan muchas transacciones de compraventa de tierras. Esto se debe, desde el punto de vista de la demanda de tierra, a que buena parte del precio de las mercancías agrícolas corresponde a la renta de la tierra, por lo que el tiempo de recuperación de la inversión de capital - en tanto ganancia- es prolongado en relación con otras actividades económicas. Por otro lado, para los oferentes, las alternativas de inversión son limitadas y no resultan lo suficientemente atractivas para desprenderse del patrimonio. 
Te vas a encontrar con campos vendidos de valores hasta 33.000 dólares la hectárea, pero no conozco operaciones cerradas en esos valores. [...] Ha sido muy bajo el volumen de operaciones que han habido en los últimos 4, 5 años. Ha habido, pero muy bajas, tanto porque los valores se han disparado así como también las locaciones de los campos, que están volviendo recién este año a retomar sus valores "semihistóricos» por hectárea en función de que han disminuido los inversores. (OI, operador inmobiliario)

En otro testimonio, un administrador agropecuario analiza cuáles pueden ser los motivos que lleven a que haya pocas transacciones de compraventa de tierras entre los productores. En base a su experiencia, el productor agropecuario de pequeña y mediana escala tampoco puede desarrollar otra opción empresarial para concretar una inversión alternativa.

[Hoy no se vende] porque no saben qué hacer con la plata. Una pregunta que te hace el productor mediano o el chico es: «Si yo vendo, ¿qué hago con la plata?» [...] La tierra es muy demandada entre comillas, la tierra vale lo que no te va a dar, no te da esa renta, pero, ¿donde la ponen? ¿En el Banco Nación?, ¿en el Banco Provincia? ¿La mandan a Suiza? Solo se venden los campos por problema de deuda o empresas grandes que tienen otras alternativas de producción. (AA, administrador agropecuario)

El incremento en el precio de la renta de la tierra se puede observar en el aumento del precio de los arrendamientos (se producen muchas más operaciones de este tipo que de compraventa), que, en el partido Junín, alcanza a 17 quintales de soja o 450 dólares por hectárea para el año 2014 (Compañía Argentina de Tierras, 2015).

\section{Los arreglos intrafamiliares en los procesos sucesorios}

El proceso sucesorio en el caso que se presenta en este artículo incluye tanto «la transferencia legal de la propiedad de la tierra y de los activos existentes», como «la transferencia a la próxima generación del uso del patrimonio y de la gerencia de la explotación» (Dirven, 2002).

De este modo, es posible distinguir dos etapas principales del proceso sucesorio, que presentan, a su vez, diferentes tipos de arreglos intrafamiliares ${ }^{5}$. Primero, se encuentra una etapa en que el productor comienza a compartir espacios de gestión de la explotación con su descendencia o parte de ella. Al principio de esta etapa, uno de los arreglos informales posibles es el otorgamiento de una remuneración a los hijos (o al único hijo) que se incorporan al trabajo de la unidad. Transcurrido un tiempo, se desarrollan otros tipos de

5. Se entiende por arreglos los distintos tipos de acuerdos que se establecen entre los diferentes miembros de la familia, tanto durante la gestión de la unidad agropecuaria para tomar las decisiones productivas y laborales y distribuir las ganancias generadas como para repartir el patrimonio familiar. 
arreglos tendientes a compartir los espacios de decisión de la empresa, como la formación de sociedades entre padres e hijos (sociedades de responsabilidad limitada, sociedades anónimas o sociedades de hecho). También se registran casos en que uno de los hijos se va incorporando al trabajo de la unidad sin recibir un pago, hasta la conformación de la sociedad. Asimismo, existen unidades en que ningún hijo está dispuesto a quedarse trabajando en la unidad, lo cual trunca este proceso. En estos casos, el padre titular del establecimiento suele convertirse en un propietario rentista, con lo que abandona su condición de productor.

En una segunda etapa, se debe resolver cómo se produce la transferencia legal de la propiedad de la tierra. En estos casos, se observa más apego a la ley para la distribución de la herencia entre los hijos y/o las hijas. Toda sucesión hereditaria comprende, dentro de las estipulaciones legales, dos fracciones: la legítima, que corresponde a los herederos forzosos, y la disponible — actualmente una quinta parte-, sobre la cual el testador puede manifestar su voluntad de destino, respetando las estipulaciones del Código Civil (artículo 3280).

El proceso sucesorio comienza mucho antes del reparto de la propiedad de la tierra, ya que lo que se encuentra en juego son los ingresos provenientes de la actividad que, por las condiciones mencionadas más arriba, han alcanzado niveles históricos. Si bien estos ingresos se componen de la tasa de ganancia de la actividad y de la renta de la tierra, se calcula que, en la actualidad, la renta de la tierra es mayor que la ganancia de la actividad agrícola, de la cual, además, deberían descontarse los costos de los insumos y de la mano de obra (Iñigo Carrera, 2007). Esta información acerca de los precios promedio en los que se puede arrendar una hectárea está muy difundida en el partido y distintas fuentes nos lo confirman. La hija de un productor que no se dedica a la producción nos comenta: "Con un rinde promedio en esta zona puede tener 35 quintales y 17 se los lleva solamente el alquiler» (L, hija de productor agropecuario). Esta información es confirmada por un operador inmobiliario de la zona: «Se han cerrado alquileres hasta en 17 quintales de soja por hectárea (a precio lleno de pizarra) y podes hablar de 11, 12 quintales en algunos sectores. Pero depende de la ambición y de los tiempos» (OI, operador inmobiliario).

En esta etapa anterior al reparto definitivo del patrimonio entre los herederos, se debe resolver la distribución de la renta de la tierra y de las ganancias y la organización de la gestión de la unidad agropecuaria, tanto cuando los hijos y/o las hijas comienzan a participar en la unidad productiva bajo las órdenes del padre o productor, como cuando pasan a hacerse cargo de la gestión de la empresa agropecuaria.

La dedicación de los productores a las tareas administrativas y de gestión de la unidad, en detrimento de las tareas físicas que implicaba el trabajo agropecuario, llevaron a que se prolongara la cantidad de años en que los padres o productores seguían al frente de la explotación, lo cual retrasó el momento de la herencia definitiva y del relevo generacional, pero también generó ins- 
tancias intermedias para incorporar a los hijos en la empresa familiar. Como explica Mazorra Paniagua (1999) para el caso español, hay una mayor permanencia del titular al frente de la explotación y una prolongación del «tiempo de espera» del sucesor para efectivamente dirigir la explotación, lo que tiene como consecuencia una mayor complejidad en los procesos de sucesión y en las relaciones intergeneracionales que afectan a la composición del trabajo y a su remuneración.

Asimismo, en esta misma etapa, muchos de los hijos y de las hijas de los productores comienzan a desarrollar sus propias trayectorias laborales. El hecho de que desarrollen trayectorias más individuales afecta a las estrategias hereditarias. En muchas ocasiones, los hijos se insertan laboralmente en el mercado después de haber seguido una carrera universitaria, y esto pone en cuestión el concepto de «hereda la tierra quien la trabaja» (Neiman, 2013) ${ }^{6}$ y da lugar al surgimiento de futuros "herederos no rurales».

En ese "tiempo de espera», se dan situaciones intermedias en las que se pueden encontrar los diferentes tipos de arreglos monetarios entre los padres y los hijos que se quedan trabajando en la unidad - pago de un monto en dinero, porcentaje de la producción y formación de sociedades-, además, también surgen los mayores conflictos entre generaciones; especialmente cuando se empieza a tener que compartir espacios de toma de decisión, tanto a nivel productivo como en la distribución de las ganancias. Estos arreglos temporales sirven para prorrogar el momento en que se produce el relevo generacional y la distribución definitiva de la herencia entre los diferentes hermanos (Neiman, 2008).

De este modo, una de las modalidades de comenzar este proceso sucesorio y transferir ingresos de la unidad productiva a los familiares antes de que se produzca la herencia definitiva es la remuneración a los trabajadores familiares. Cuando los hijos son jóvenes y quieren participar en el trabajo de la explotación, lo hacen en un lugar subordinado a las decisiones del padre productor. En estos casos, el pago puede tener una forma salarial — con una remuneración mensual-, también es posible percibir un porcentaje de la producción en el momento de vender la cosecha o hay veces que se les permite desarrollar una actividad asociada con la unidad agropecuaria, como, por ejemplo, prestar servicios de maquinaria con los equipos de la unidad o desarrollar una actividad independiente en una fracción del predio. En estos procesos, el hijo que trabaja en la explotación y recibe un pago será, probablemente, el encargado de ocuparse en el futuro de la gestión de la unidad. A continuación, se presenta el testimonio de una esposa de productor que cuenta cómo se integró recientemente el hijo al trabajo agropecuario.

6. El alto nivel de escolaridad alcanzado por los hijos de productores se puede verificar, ya que todos los hijos de productores de más de 13 años accedieron, por lo menos, al nivel secundario o al polimodal. Asimismo, el 34,6\% de los hijos de 19 a 25 años y el $40,3 \%$ de los mayores de 25 años alcanzaron, incluso, niveles terciarios o universitarios (Neiman, 2011). 
En el campo, trabajan mi marido y mi hijo varón de 18 años, que, como decidió no estudiar al terminar el polimodal, trabaja en el campo recibiendo una remuneración de 500 pesos semanales [este es el primer año que trabaja] [...] Él opera las maquinarias y realiza todo el resto de las tareas que también hace el padre. (M, casada, esposa de productor)

En un segundo momento de esta etapa, los arreglos familiares entre la generación de los padres y la de los hijos toman la forma de una sociedad familiar, ya sea en términos formales, en las que se encuentra estatuida en porcentajes fijos la forma en que se reparten las ganancias, o como sociedades informales, en las que el padre pone el recurso - tierra y maquinaria- y los hijos, el trabajo. En estos casos, se debe decidir en familia cómo se reparten las ganancias y cómo se toman las decisiones productivas y organizativas. Sin lugar a dudas, este es el momento de mayores tensiones, porque por primera vez se empiezan a proponer cambios en la organización de la producción y de la familia.

Si bien, por un lado, la formación de sociedades entre diferentes miembros de la familia resuelve cuestiones vinculadas con la organización de la unidad familiar agropecuaria, por otro lado, también responde a cuestiones administrativas y contables:

Normalmente, en caso de que el viejo ya deje la explotación o que, por una cuestión impositiva, involucre a los hijos, se hacen sociedades. Se hacen sociedades anónimas o SRL por una cuestión impositiva y, si no, se hacen sociedades de hecho, pero como las sociedades de hecho no transfieren los bienes, queda el bien a nombre de una persona, que antes de fallecer o cuando ya la persona es grande y quiere desprenderse de la explotación, la dona a los hijos. (E, escribano)

Gilding (2005), en su trabajo sobre empresas familiares australianas, explica que la política impositiva también puede promover la formación de empresas familiares en búsqueda de una minimización en sus impuestos, e incluye el siguiente testimonio de un empresario: "Have any assets at all, apart from the clothes I've got on and a few things» ${ }^{7}$. Con esta misma lógica, en el caso en estudio, la formación de sociedades familiares — como sociedades anónimas o sociedades de responsabilidad limitada — apunta a transferir riesgos, disminuir cargas impositivas y proteger la propiedad.

El caso que se presenta a continuación es el de un pequeño productor ( 45 hectáreas) que, además, vive en la misma explotación agropecuaria. Este decidió establecer una sociedad con el hijo que continuó trabajando en la unidad agropecuaria.

Tengo dos varones y una nena. La mayor ya está casada y tiene dos hijos, vive en Junín. Los otros dos viven acá, en el campo. El que trabaja en el campo y

7. Traducción: «Aparte de mi ropa y de algunas pocas cosas, no tengo ningún otro activo». 
es pago es el menor (30 años), con el que figuramos como una sociedad de hecho. La sociedad la formamos cuando yo me jubilé y yo me quedo con el 60\% y mi hijo, el 40\%. [...] Las decisiones de qué producir las tomamos un poquito los dos juntos. Ahora ya casi las toma ya más él que yo. Uno ya tiene gana de andar retirándose un poco y alguien tiene que seguir. (P, 70 años, viudo, productor de 45 hectáreas)

Al tratarse de una explotación pequeña, la residencia de uno de los hijos en la propiedad tiene un peso importante al decidir el tipo de sociedad que se constituye, aunque no haya aún un traspaso definitivo de la propiedad.

La formación de este tipo de sociedades responde a una necesidad de resolver la gestión de la explotación agropecuaria con la participación de dos generaciones durante el "tiempo de espera» a la sucesión definitiva de la propiedad. De esta manera, se distribuyen los ingresos provenientes de la renta de la tierra, del trabajo en la unidad y de las ganancias de la actividad agropecuaria. Además, se busca aliviar cargas impositivas y transferir algunos riesgos del propietario de la tierra a la sociedad constituida para llevar a cabo la actividad económica.

En una segunda etapa del proceso sucesorio, se produce, después del fallecimiento del padre productor, el reemplazo en la gestión de la explotación y la transferencia de la propiedad. Como fue explicado anteriormente, existe un marco legal de acuerdo con el cual se realiza la sucesión de la tierra. Sin embargo, se vuelven a establecer nuevos arreglos familiares para la nueva gestión de la unidad productiva.

A continuación, se presentan dos testimonios de dos hijas de productores que han fallecido que dan cuenta de este tipo de arreglos. En el primer caso, ella estableció una sociedad de hecho ${ }^{8}$ para la explotación del campo familiar con su hermano y su madre. En sus palabras, se organizan del siguiente modo:

[...] nosotros le alquilamos a las dueñas del campo, que son mi mamá, mis tías y mi abuela. Pagamos un alquiler como si fuéramos un arrendatario más. [...] Yo estoy en la sociedad pero no trabajo ahí. (L, 40 años, casada, hija de productora de 280 hectáreas, padre fallecido)

Aquí se puede distinguir entre ser socio y participar en las ganancias de la empresa; ser propietario de la tierra y percibir un canon de arrendamiento, o ser socio y además trabajar en la empresa a cambio de un salario.

El segundo caso es muy similar, aunque el único que se encarga de la explotación de la unidad productiva es el hijo varón, que paga un canon de arrendamiento a su hermana y a su madre.

8. La sociedad de hecho es la sociedad más sencilla. No está constituida regularmente, es decir, no adopta uno de los tipos específicos de la ley y no tiene un contrato social, por lo que tampoco está inscripta en el Registro Público de Comercio, pero tiene como ventaja sus bajos costos de constitución y ciertos beneficios impositivos. 
Ahora, en este momento, mi hermano nos pasa un monto en quintales de soja, como si fuera un alquiler, pero, por ejemplo, cuando se complica con la parte impositiva, no tengo ningún problema en que a mí me baje el alquiler, en ese sentido, a mí no me molesta. [...] Mientras viva mi mamá, va a seguir así. Después, no sé. (AH, 42 años, soltera, hija de productora de 400 hectáreas, padre fallecido)

En este caso, se observa también que el contexto familiar de los acuerdos puede otorgar algo de flexibilidad a los arreglos contractuales respecto a los valores de arrendamiento.

El otro tipo de arreglo presente en estas unidades parentales es la remuneración al trabajador familiar que se ocupa de la gestión de la sociedad constituida para llevar a cabo la actividad agropecuaria. Estos son los casos en que el padre productor ha fallecido o se ha retirado de la actividad y se ha formado una sociedad entre los hijos y/o las hijas o entre los hijos, las hijas y la madre. Se suele pagar un canon por la renta de la tierra a cada uno de los miembros de la sociedad, pero, además, los trabajadores familiares (en general, el hijo varón) cobran una remuneración por ocuparse de llevar a cabo las tareas necesarias para la actividad. Esta es la forma en que se organiza la sociedad integrada por los dos hermanos y la madre que se presentó anteriormente, en la cual el hijo varón y su madre trabajan en la gestión de la actividad.

Mi mamá hace más la parte de bancos y la numérica y la planificación, y mi hermano está en la ejecución diaria. Mi hermano tiene un sueldo y mi mamá también. Yo no, yo no tengo sueldo. Después, si hay dividendos, se reparten, que casi nunca hay, porque siempre se invierte. (L, 40 años, casada, hija de productora de 280 hectáreas, padre fallecido)

De esta forma, se pueden distinguir, analíticamente, los ingresos provenientes de la renta de la tierra, que toman la forma de arrendamientos intrafamiliares a precios de mercado, o "a precios familiares», de aquellos que forman parte de la ganancia de la actividad, de los que se les debe descontar los insumos y la remuneración a los trabajadores no familiares y familiares, ya sea mediante el otorgamiento de un pago mensual o un porcentaje mayor en el momento del reparto de las ganancias entre los miembros de la sociedad.

El siguiente esquema resume las características que asume el modelo de sucesión en las pequeñas y medianas empresas de esta zona núcleo de la región pampeana que ha atravesado un período de auge económico. En una lectura vertical, del lado izquierdo, están registradas las etapas del proceso hereditario y, en el lado derecho, aparecen la diversidad de arreglos familiares que se corresponden con las etapas mencionadas y sus principales particularidades. 
Esquema. Modelo de sucesión en la pequeña y mediana empresa agrícola familiar pampeana

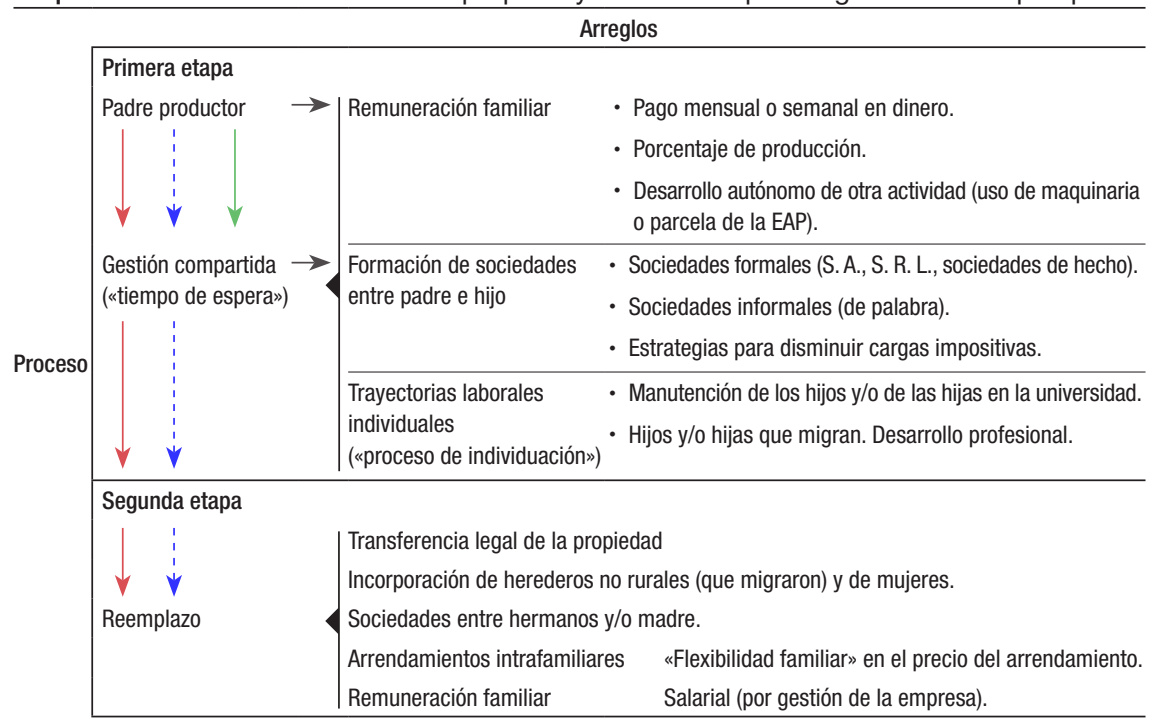

\section{Conclusiones}

El incremento del precio de la tierra y de la producción agropecuaria interviene en la formulación de arreglos entre los miembros de la familia para llevar a cabo el proceso hereditario. Así, aparecen variados arreglos familiares en torno al acceso a la propiedad y a los beneficios económicos de la producción durante dicho proceso.

La importancia de comprender los aspectos que conforman el precio de la tierra y de la producción permite diferenciar la renta, la ganancia y el salario como partes del ingreso total de la explotación. Estos factores se distribuyen de distinto modo según los arreglos entre los diferentes actores, ya sean familiares o no familiares.

Entre los arreglos familiares anteriores a la sucesión definitiva, puede haber una gran variedad de situaciones, dentro de las cuales se encuentra difundida la formación de sociedades familiares que arriendan la tierra al padre o a otro pariente como parte de una estrategia económico-impositiva, y la remuneración familiar, ya sea del padre al hijo o entre hermanos y/o hermanas. Los ingresos obtenidos a partir de este arrendamiento intrafamiliar se identifican como provenientes de la renta de la tierra, que, como se ha visto, presenta precios de mercado que pueden flexibilizarse en determinadas circunstancias por el vínculo de sangre que media en el arreglo. Los ingresos que percibe un trabajador familiar por su trabajo en la explotación agropecuaria toma la forma de salario, ya sea percibiendo un valor mensual o un porcentaje de la producción. El resto de los ingresos de la sociedad familiar es invertido en concepto de insumos para la reproducción de la unidad productiva, salarios para trabajadores extraparentales y ganancias de la sociedad. 
Los arreglos descriptos muestran el devenir de un proceso hereditario marcado por una primera etapa de una gestión de la empresa compartida entre un padre productor y un hijo (generalmente, varón), y una segunda etapa en la que los familiares del productor (hijos, y/o hijas y, a veces, esposa) se deben hacer cargo de la unidad, con el consecuente reparto de tareas y de ingresos.

La introducción del trabajo de profesionales (abogados, contadores o escribanos) en los procesos sucesorios y en la conformación de sociedades muestra la intención de optimizar la organización de la empresa agropecuaria en términos económicos, considerando el encuadramiento legal respectivo. Esto también da cuenta del carácter empresarial y capitalista de dicho tipo de unidad productiva.

Se observa cierta planificación familiar en torno al modo cómo se transita por este proceso que, por lo menos desde el aspecto discursivo, apunta a garantizar la equidad distributiva entre los hijos y/o las hijas y a mantener la propiedad indivisa. Asimismo, se puede ver que ningún sucesor o sucesora está dispuesto a renunciar a la herencia de la propiedad de la tierra, ya sea en virtud del género, por haber migrado o por haber abandonado la actividad (presencia de herederos no rurales). Sin embargo, es posible distinguir una etapa anterior a los arreglos para el reparto de la propiedad en la que se hereda la gestión de la empresa agropecuaria. En esta etapa, es más común encontrar a un hijo varón asumiendo el rol de sucesor en la dirección de la unidad, reciba a cambio un salario o una participación en la sociedad con el padre productor.

En este tipo de unidades, los familiares suelen desvincularse del trabajo en la unidad económica, tanto por las características de la actividad (expulsora de mano de obra) como por los procesos de individuación que atraviesan los hijos y/o las hijas que acceden a altos niveles de formación y que se insertan en el mercado de trabajo como profesionales. Estos procesos pueden alejarlos (a todos o a algunos de ellos), en un primer momento, de la gestión de la empresa familiar sin que esto signifique renunciar a la herencia de la propiedad. En lo referido a la sucesión patrimonial definitiva, la herencia de la propiedad se termina, en última instancia, circunscribiendo a lo indicado en el marco legal vigente. De esta forma, se minimiza el reparto discrecional de la tierra en función de características tales como el género, la residencia o la profesión de los hijos.

El modelo de sucesión estudiado se inscribe en la discusión acerca del papel de la herencia y de "la familia» como institución en el desarrollo del capitalismo. La herencia fue considerada tempranamente por la sociología como un concepto clave para entender la organización en clases de la sociedad y su permanencia a través del pasaje de privilegios de una generación a otra y la conservación del «linaje». Sobre estos fundamentos, se incorporó, durante el siglo Xx, el debate sobre la relación que se establece entre familia y empresa en el desarrollo y en la especialización de las relaciones capitalistas de producción. En tanto algunas perspectivas coincidían en la centralidad de este vínculo, otras señalaban que la creciente especialización conducía al gerenciamiento y al funcionamiento en red de las empresas, dejando a la familia en un segundo plano de la gestión. 
La organización familiar en torno a la herencia en las empresas familiares agropecuarias de esta región particular de la Argentina muestra la centralidad de la familia como sujeto protagónico del desarrollo agrario, involucrado en la actualidad en un período de innovación tecnológica y de auge económico de la actividad. En este escenario, el modelo hereditario garantiza la continuidad de las empresas agropecuarias con bajos niveles de subdivisión o de venta de la tierra. Asimismo, si bien las características de la actividad impulsan la desvinculación de la familia de las labores de la explotación, el rendimiento económico y el precio de la tierra llevan a que ninguno de los hijos o de las hijas resigne su participación en la herencia. Las diferencias de género, de profesión o de primogenitura no aparecen como legítimas para que una parte de la descendencia decida renunciar a su posición de clase propietaria ni a las consideraciones que lleva asociadas.

Los resultados alcanzados en este artículo contribuyen a caracterizar y a conceptualizar acerca de la evolución del sector agrario pampeano y de un actor social y político históricamente clave en el desarrollo agropecuario argentino, aunque desde una perspectiva menos frecuentada. Estos avances tienen una implicación práctica para el diseño de políticas de desarrollo regional y para orientar futuras investigaciones que apunten a la articulación entre las características que asumen las empresas familiares en el desarrollo del capitalismo y las formas de participación económica y de representación política.

\section{Referencias bibliográficas}

Aвramovay, R. (2001). Os impasses sociais da sucessoa hereditária na agricultura familiar. Florianópolis: EPAGRI; Brasilia: NEAD. Ministério do Desenvolvimento Agrário.

Anderson, R. y Rosenblatt, P. (1985). «Intergenerational transfer of farm land». Journal of Rural Community Psychology, 6, 19-25.

Archetti, E. y StÖLen, K.A. (1975). Explotación familiar y acumulación de capital en el campo argentino. Buenos Aires: Siglo XXI.

Baptista, F. O. (1995). «Agriculture, rural society and the land question in Portugal». Sociologia Ruralis, 35: 309-321. <http://dx.doi.org/10.1111\%2Fj.1467-9523.1995.tb00841.x> .

BARDOMÁs, S. (2000). "Trayectorias en la agricultura familiar: Tierra, producción y herencia en Pigüé (1920-1994)». Documentos de trabajo CEIL-PIETTE. Buenos Aires: CEIL-PIETTE.

Barthez, A. (1990). «Familia, actividad y pluriactividad en la agricultura». En: Arkleton Research. Cambio rural en Europa. Madrid: Ministerio de Agricultura, Pesca y Alimentación, 161-178.

Bennett, J.W. (1982). «The agrifamily system». En: Bennett, J.W. Of time and the enterprise. Minneapolis, MN: University of Minnesota Press, 128-147.

Berle, A. y Means, G.C. (1932). The Modern Corporation and Private Property. Nueva York: World Inc.

Burton, R. y Walford, N. (2005). «Multiple succession and land division on family farms in the South East of England: A counterbalance to agricultural concentration?». Journal of Rural Studies [en línea], 21, 335-347. $<$ http://dx.doi.org/10.1016/j.jrurstud.2005.04.004>. 
Chayanov, A. (1975). La organización de la unidad económica campesina. Ciudad de México: Cultura Popular.

Colli, A. y Rose, M. (1995). «Family Business». En: Rose, M.B. Family Business. Aldershot: Edward Elgar Publishing.

Colli, A.; Pérez, P. y Rose, N. (2003). «National Determinants of Family Firm Development?: Family Firms in Britain, Spain, and Italy in the Nineteenth and Twentieth Centuries». Enterprise \& Society [en línea], 4 (1), 28-64. <http://dx.doi.org/10.1017/s1467222700012441>.

Compañía Argentina de Tierras (2015) [en línea]. Buenos Aires. <http://www. cadetierras.com.ar/>.

Cooney, T.M. y Dykstra, P. (2011). «Family obligations and support behavior: A United States-Netherland comparison». Ageing Soc. [en línea], 31 (6), 1026-1050. <http://dx.doi.org/10.1017/s0144686x10001339>.

Craviotti, C. (2001). "Tendencias en el trabajo agrario y dinámicas familiares». $V$ Congreso Nacional de Estudios del Trabajo. Buenos Aires: ASET.

Danes, S. y Amarapurkar, S. (2001). "Business tensions and success in farm family businesses». Family Economics and Resource Management Biennial, 4, 178-190.

Danes, S. y LeE, Y. (2004). "Tension generated by business issues in farm businessowning couples». Family Relations, 53 (4), 357-366. <http://dx.doi.org/10.1111/j.0197-6664.2004.00042.x>.

Danes, S. M., Leichtentritt, R., Metz, M. E., \& Huddleston-Casas, C. (2000). "Effects of conflict styles and conflict severity». Journal of Family and Economic, 2, 259-286. <http://dx.doi.org/10.1023/a:1009485301715>

Danes, S. y Olson, P.D. (2003). "Women's involvement in family businesses, business tensions, and business success». Family Business Review [en línea], 16, 53-68. <http://dx.doi.org/10.1111/j.1741-6248.2003.00053.x>.

Dirven, M. (2002). «Las prácticas de herencia de tierras agrícolas: ¿Una razón más de éxodo de la juventud?». Serie Desarrollo Productivo, 135. CEPAL: Santiago de Chile.

Donnelly, R.G. (1964). "The Family Business». Harvard Business Review, 42 (4), 93-105.

Dunn, M. (1980). "The Family Office: Coordinating Mechanism of the Ruling Class». En: Domнoff, G.W. (ed.). Power Structure Research [en línea]. Beverly Hills, CA: Sage, 17-44. <http://dx.doi.org/10.1177/089692058000900202>.

EtXeZARRETA, M. et al. (1995). La agricultura familiar ante las nuevas políticas agrarias comunitarias. Madrid: MAPA.

Fortes, M. (1958). «Introduction». En: Goody, J. (ed.). The developmental cycle in domestic groups. Cambridge: Cambridge University Press. Cambridge Papers in Social Anthropology, 1.

Gasson, R. et al. (1988). "The farm as a family business: A review». Journal of Agricultural Economics [en línea], 39, 1-41.

<http://dx.doi.org/10.1111/j.1477-9552.1988.tb00560.x>.

Gilding, M. (2005). "Families and fortunes: Accumulation, management succession and inheritance in wealthy families». Journal of Sociology [en línea], 41 (1), 29-45. <http://dx.doi.org/10.1177/1440783305050962>.

GonzÁlez, J. y Gómez Benito, C. (1997). «Clases agrarias, estrategias familiares y mercado de trabajo». En: Agricultura y Sociedad en la España contemporánea. Madrid: Ministerio de Agricultura, Pesca y Alimentación, 565-580. 
Gras, C. (2010). «La agricultura familiar en el agro pampeano: Desplazamientos y mutaciones». En: Cerdá, J. y Gutiérrez, T. (comp.). Trabajo agrícola: Experiencias y resignificación de las identidades en el campo argentino. Buenos Aires: CICCUS.

Gutman, G. et al. (1983). El sector agropecuario argentino: Una estimación de los excedentes en el periodo 1950-1982. Buenos Aires: CFI.

Hutson, J. (1987). "Fathers and Sons: Family Farms, Family Businesses and the Farming Industry». Sociology [en línea], 21 (2), 215-229. <http://dx.doi.org/10.1177/0038038587021002004>.

INDEC (1988). Censo Nacional Agropecuario. Buenos Aires.

- (2002). Censo Nacional Agropecuario. Buenos Aires.

Iñigo Carrera, J. (2007). La formación económica de la sociedad argentina. Vol. I, Renta agraria, ganancia industrial y deuda externa. 1882-2004. Buenos Aires: Imago Mundi.

Jervell, A. (1999). "Changing patterns of family farming and pluriactivity». Sociologia Ruralis [en línea], 39 (1), 100-116. <http://dx.doi.org/10.1111/1467-9523.00095>.

Kautsky, K. (1978). La cuestión agraria. México: Ediciones Cultura Popular.

Keating, N.; Fast, J.; Lero, D.; Lucas, S. y Eales, J. (2014). "A taxonomy of the economic costs of family care to adults». Elsevier [en línea], 3 (abril), 11-20. $<$ http://dx.doi.org/10.1016/j.jeoa.2014.03.002>.

Keating, N. y Little, H. (1997). "Choosing the Successor in New Zealand Family Farms». Family Business Review [en línea], 10 (2), 157-171. <http://dx.doi.org/10.1111/j.1741-6248.1997.00157.x>.

Keating, N. y Munro, B. (1989). «Transferring the family farm: Process and implications». Family Relations [en línea], 38, 215-218. <http://dx.doi.org/10.2307/583678>.

Keller, M. (2008). "Argentina, China, y las oleaginosas». Revista Alimentos Argentinos, 43.

«Los campos de la zona valen cada vez más». La Democracia [en línea], 2014. <http://www.diariodemocracia.com/notas/2011/5/25/locales-25496.asp>.

Levinson, H. (1971). "Conflicts that plague family businesses». Harvard Business Review, 49(2), 90-98.

Mann, S. (1990). Agrarian capitalism in theory and practice. The University of North Carolina Press.

Márgenes Agropecuarios [en línea]. <http://www.margenes.com/>.

Marx, K. (1976). El capital, crítica de la economía política. Bogotá/México: FCE, I, II y III.

Mazorra Paniagua, A. (1999). "La remuneración del trabajo en la agricultura familiar española (1985-1997)». Investigaciones Geográficas, 22, 129-148.

McClendon, R. y Kadis, L.B. (1991). «Family therapists and family business: A view of the future». Contemporary Family Therapy [en línea], 13, 641-651. <http://dx.doi.org/10.1007/bf00890598>.

McCullom, M.E. (1988). «Integration in the family firm: When the family system replaces controls and culture». Family Business Review [en línea], 1, 399-417. <http://dx.doi.org/10.1111/j.1741-6248.1988.00399.x>.

MINAGRI (2016). Ministerio de Agricultura, Ganadería y Pesca: Sistema Integrado de Información Agropecuaria [en línea]. Buenos Aires: MAGyP-DMA. <http://www. agroindustria.gob.ar> [Consulta: 1 febrero 2016]. 
Muzlera, J. (2009). «Estructura social, mercado de trabajo y sociabilidad de los pueblos rurales del sur santafecino a comienzos del siglo xxi: Una mirada intraregional a la heterogeneidad del "nuevo modelo" agropecuario». En: Cerdá, J. y GutiéRREZ, T. (comp.). Trabajo agrícola: Experiencias y resignificación de las identidades en el campo argentino. Buenos Aires: CICCUS.

Neiman, M. (2008). La organización del trabajo en la agricultura familiar. Los hogares con trabajadores familiares remunerados en la región pampeana: El caso del Partido de Junín. Buenos Aires: FLACSO. Tesis de maestría en Estudios Sociales Agrarios.

- (2011). La agricultura familiar en la región pampeana: Nuevos vínculos familiares y transformaciones en las unidades de producción. Buenos Aires: Facultad de Ciencias Sociales. Universidad de Buenos Aires. Tesis de doctorado en Ciencias Sociales.

- (2013). «La herencia: los/as hijos/as y el tránsito entre generaciones en la agricultura familiar de la región pampeana argentina». Revista de Estudios Sociológicos, XXXI (93), 899-920.

Nosé, L.; Korunka, C.; Frank, H. y Danes, S. (2015). «Decreasing the Effects of Relationship Conflict on Family Businesses: The Moderating Role of Family Climate». Journal of Family Issues [en línea], 25 (febrero), 1-27. <http://dx.doi.org/10.1177/0192513x15573869>.

RaGin, C. (2007). «El uso de los métodos comparativos para estudiar la diversidad». En: La construcción de la investigación social. Bogotá: Siglo del Hombre.

Rettig, K.; Leichtentritt, R. y DAnes, S. (1999). "The effects of resources, decision making, and decision implementing on perceived family well-being in adjusting to an economic stressor». Journal of Family and Economic Issues, 20, 5-34.

Robichaux, D. (2007). Familia y diversidad en América Latina: Estudios de casos. Buenos Aires: CLACSO (Consejo Latinoamericano de Ciencias Sociales).

Rodríguez, J. y ArCeO, N. (2006). "Renta agraria y ganancias extraordinarias en la Argentina 1990-2003». Realidad Económica, 219.

Rogers, S. y SALAmon, S. (1983). "Inheritance and social organization among family farmers». American Ethnologist [en línea], 10, 529-548. <http://dx.doi.org/10.1525/ae.1983.10.3.02a00080>.

Sistema Integral de Información Agropecuaria (SIIA). Programa de Servicios Agricolas Provinciales [en línea]. Buenos Aires: Ministerio de Agricultura, Ganadería y Pesca de la Nación. <www.siia.gov.ar>.

Stewart, C. y Danes, S. (2001). «The relationship between inclusion and control in resort family businesses: A developmental approach to conflict». Journal of Family and Economic Issues, 22, 293-320.

Taylor, J.; Norris, J. y Howard, W. (1998). «Succession patterns of farmer and successor in Canadian farm families». Rural Sociology [en línea], 63, 553-573. <http://dx.doi.org/10.1111/j.1549-0831.1998.tb00692.x>.

Valle Baeza, A. (1991). Valor y precio: Una forma de regulación del trabajo social. Ciudad de México: Universidad Autónoma de México.

Wolf, E. (1978). Los campesinos. Madrid: Nueva Visión Labor. Zeituin, M. (1989). The Large Corporation and Contemporary Classes. New Brunswick, NJ: Rutgers University Press. 